\title{
Next-Generation Sequencing in Order to Better Characterize a BRCA Variant of Uncertain Significance
}

\author{
Steven Sorscher ${ }^{\mathrm{a}} \quad$ Shakti Ramkissoon ${ }^{\mathrm{b}}$ \\ ancology Division, Wake Forest School of Medicine, Winston-Salem, NC, USA; \\ ${ }^{b}$ Foundation Medicine, Inc., Cambridge, MA, USA
}

\section{Keywords}

BRCA variant · Variant of uncertain significance $\cdot$ Next-generation sequencing

\begin{abstract}
BRCA germline mutations are the most common predisposing factor in familial breastovarian cancer syndrome families. However, many screened patients are identified as harboring BRCA variants of uncertain significance (VUS), rather than carrying deleterious germline mutations [Calo et al.: Cancers 2010;2:1644-1660]. While such VUSs are typically reclassified as benign polymorphisms, this may occur years after the VUS is first identified [Murray et al.: Genet Med 2011;13;998-1005]. Loss of heterozygosity (LOH) of BRCA is nearly always the gatekeeper event in inherited BRCA-related breast cancer and LOH of BRCA is rare in sporadic cancers [Osorio et al.: Int J Cancer 2002;99:305-309]. Here, we describe a patient identified as carrying a germline BRCA VUS. Tumor next-generation sequencing (NGS) demonstrated a very high mutation allelic frequency for that BRCA VUS, consistent with LOH. This case illustrates that since BRCA LOH is the typical mechanism of transformation in inherited BRCArelated breast cancers, NGS might be used to suggest that the BRCA VUS is actually cancer predisposing in a particular family. As a result, this may help patients make more informed decisions regarding screening and prophylactic therapy, long before official reclassification of the VUS occurs.


 Oncology}

Sorscher and Ramkissoon: Next-Generation Sequencing in Order to Better Characterize a BRCA Variant of Uncertain Significance

\section{Introduction}

Screening patients for germline deleterious BRCA mutations has become very common. Rather than deleterious mutations, patients may be identified as harboring a germline VUS. Although the percentage of screened patients identified as harboring a BRCA variant of uncertain significance (VUS) varies with the pretest probability of the population studied (ascertainment bias), an overall BRCA VUS rate of $7-15 \%$ has been reported for screened patients [1]. While many factors have been proposed for reclassification of a VUS as a deleterious versus a benign polymorphism, co-segregation in families harboring the particular VUS has been the primary basis for reclassification used in genetic counseling [1, 2]. However, reclassification can take many months or years to occur from the time of the VUS being identified [3]. This leaves patients uncertain as to the usefulness of increased screening measures and prophylactic treatments (including surgery) while awaiting reclassification.

Here we describe a patient identified as harboring a BRCA2 VUS (N1758D). Next-generation sequencing (NGS) of her tumor showed the BRCA2 VUS mutation allelic frequency (MAF) to be very high, consistent with BRCA2 loss of heterozygosity (LOH). LOH is very rare in sporadic breast cancers [4-6]. Also, roughly 93\% of breast cancers related to BRCA germline mutations demonstrate LOH [6]. Therefore, the LOH (high MAF) seen in our patient's tumor suggests a high likelihood that the identified VUS should in fact be considered a deleterious mutation, rather than a benign polymorphism.

Germline testing has become far more routine in the last few years for patients concerned with the possibility of carrying a germline BRCA gene mutation associated with the familial breast-ovarian cancer syndrome, and NGS of tumors is nearly routine for patients hoping to pursue targeted therapies based on identified actionable molecular abnormalities. Based on the apparently high sensitivity and specificity of BRCA2 LOH related to familial breast-ovarian cancer syndrome, molecular information gleamed from tumor NGS may help patients make more informed decisions regarding surveillance and even prophylactic surgery once a germline VUS has been identified, and often long before enough co-segregation and other information is available for the purpose of reclassifying a particular BRCA2 VUS as a deleterious or as a benign polymorphism.

\section{Case Report}

T.T. is a 55-year-old patient who underwent a left-sided mastectomy in January 2013 for a T1cNoMO or stage 1 breast cancer $(1.8 \mathrm{~cm}$ invasive ductal carcinoma, ER/PR positive, Her2 negative for gene amplification by FISH). She discontinued adjuvant aromatase inhibitor therapy after a few months due to gastrointestinal side effects. There has been no recurrence of her breast cancer to date.

Family history significantly showed that T.T.'s mother, maternal grandmother, and two maternal aunts had breast cancer (ages unknown).

Comprehensive BRCA analysis (germline testing) showed a BRCA2 N1758D "genetic variant, favor polymorphism" (Myriad Genetics, Salt Lake City, UT, USA). That variant has not been reclassified to date.

In December 2016, NGS was used to analyze her primary tumor that was removed in 2013. Results demonstrated the same BRCA2 N1758D variant. The MAF was reported to be 84.3\% (FoundationMedicine, Inc., Cambridge, MA, USA) 
 Oncology}

\section{Discussion}

The lack of expression of normal tumor suppressor gene products is the mechanism of tumorigenesis for nearly all inherited cancer syndromes, including BRCA-related breastovarian cancer syndrome, the most common inherited breast cancer hereditary syndrome. Screening patients for BRCA germline mutations results in identifying a BRCA VUS in roughly $7-15 \%$ of patients, a rate that appears higher in the Hispanic (22\%) and African-American (46\%) populations [3].

Patients identified as harboring germline VUSs are advised that most VUSs are later classified as benign, and guidelines therefore suggest screening and prophylactic surgery considerations be based on personal and family history without regard to the VUS [7]. Reclassification of VUSs can occur long after identification and patients with VUSs often elect for aggressive screening and treatment based on the recommendations for known BRCA deleterious mutations in spite of the uncertainty related to a VUS which is awaiting reclassification. For example, Murray et al. [3] noted reclassification can occur months or years after disclosure of a particular VUS and reported a series of patients with BRCA VUSs who made variable decisions regarding surgery well before reclassification occurred.

BRCA LOH is "very rare" in sporadic breast cancers [4-6]. Loss of expression due to LOH is the mechanism of transformation in roughly $93 \%$ of cases of BRCA-related breast cancers [6]. Our patient harbored a BRCA2 VUS and the tumor demonstrated LOH (high MAF). In fact, although BRCA LOH can be seen in sporadic breast cancers, this event is so rare that patients who carry germline BRCA deleterious mutations and who develop breast or ovarian cancers are assumed to have developed the cancer due to LOH (Knudson 2-hit hypothesis). Taken together, these finding suggests that BRCA VUS LOH was the gatekeeper molecular mechanism of transformation in our patient's breast cancer.

If BRCA VUS LOH had not been demonstrated in the tumor, the BRCA VUS might still have predisposed the patient to tumorigenesis. Inactivation of the second allele might occur through somatic genomic alteration or transcriptional silencing, loss of BRCA function due to haploinsufficiency (resulting from DNA damage) or failure of posttranslational modification [8]. Thus, had NGS of the tumor not shown LOH, the patient should be informed that it remains possible that the germline BRCA VUS might still have been an important risk factor in the development of her breast cancer.

While not definitive, the molecular information derived from testing the tumor might mitigate the challenges patients face when making decisions regarding screening and prophylactic treatment. Since $\mathrm{LOH}$ is the footprint of the mechanism of tumorigenesis for patients inheriting a deleterious BRCA mutation, a patient whose tumor shows BRCA LOH might more reasonably pursue aggressive screening and prophylactic treatment, as this would be evidence of the BRCA VUS being predisposing in that patient.

Genetic counseling guidelines continue to rely predominantly on personal and family histories as the basis for reclassification of a BRCA VUS [1,2]. As more patients are screened for germline variants, more are being identified as carrying a VUS. NGS is becoming nearly routine as well. While MAF is not a validated metric from NGS testing, the obtained results were used in this patient to suggest that her BRCA VUS was likely deleterious. Together with her strong personal and family history, the molecular information might be useful in helping the patient more strongly consider screening and even prophylactic surgery options. However, the penetrance of VUSs reclassified as deleterious based primarily on co-segregation, or (as proposed in this case) based on molecular analysis of the tumor or even BRCA protein analysis, cannot be assumed to be the same as the risk for breast cancer development asso- 
ciated with patients who undergo testing based on a high pretest probability for harboring a BRCA mutation and who are found to carry a germline deleterious mutation. The clinical history - personal and family history - should remain the gold standard. Reclassifying a VUS as a deleterious mutation molecularly in a particular patient in a particular family might allow for a more individualized or personalized estimate of penetrance, as the affected and unaffected family members could harbor linked polymorphisms that increase or decrease the possibility of the transforming event, $\mathrm{LOH}$.

This case report illustrates that when tumor $\mathrm{LOH}$ is the molecular hallmark of transformation and the same $\mathrm{LOH}$ is rarely seen in sporadic cancers, identifying tumor $\mathrm{LOH}$ is strongly suggestive of the germline VUS in fact being deleterious for that patient. Such molecular information might occasionally help patients make more informed decisions regarding screening and prophylactic treatments and potentially make those decisions sooner than by awaiting reclassification based on the current standard reclassification methods.

\section{Statement of Ethics}

Drs. Sorscher and Ramikissoon have no ethical conflicts to disclose.

\section{Disclosure Statement}

Dr. Sorscher has no conflict of interest to disclose. Dr. Ramikissoon is employed by Foundation Medicine, Cambridge, MA, USA.

\section{References}

1 Calo V, Bruno L, La Paglia L, et al: The clinical significance of unknown sequence variants in BRCA genes. Cancers 2010;2:1644-1660.

-2 Richards S, Aziz N, Bale S, et al: Standards and guidelines for the interpretation of sequence variants: a joint consensus recommendation of the American College of Medical Genetics and Genomics and the Association for Molecular Pathology. Genet Med 2015;17:405-424.

3 Murray ML, Cerrato F, Bennett RL, et al: Follow-up of carriers of BRCA1 and BRCA2 variants of unknown significance: variant reclassification and surgical decisions. Genet Med 2011;13:998-1005.

4 Teng DH, Bogden R, Mitchell J, et al: Low incidence of BRCA2 mutations in breast carcinoma and other cancers. Nat Genet 1996;13:241-244.

5 Khoo US, Ozcelik H, Cheung ANY, et al: Somatic mutations in the BRCA1 gene in Chinese sporadic breast and ovarian cancer. Oncogene 1999;18:4643-4646.

6 Osorio A, de la Hoya M, Rodriguez-Lopez R, et al: Loss of heterozygosity analysis at the BRCA loci in tumor samples from patients with familial breast cancer. Int J Cancer 2002;99:305-309.

7 Daly MB, Pilarski R, Berry M, Buys SS, Farmer M, Friedman S, et al: NCCN Guidelines Insights: Genetic/familial high-risk assessment: breast and ovarian, version 2.2017. J Natl Compr Canc Netw 2017;15:9-20.

-8 Welcsh PL, King MC: BRCA1 and BRCA2 and the genetics of breast and ovarian cancer. Hum Mol Genet 2001;10:705-713. 